\title{
Detecting the organization of materials: Perceiving the forest despite the trees
}

\author{
MELISSA J. GUYNN and GILLES O. EINSTEIN \\ Furman University, Greenville, South Carolina \\ and \\ R. REED HUNT \\ University of North Carolina, Greensboro, North Carolina
}

\begin{abstract}
Relational processing refers to encoding relations among items. Individual-item processing refers to encoding information that makes items discriminable from other items. The present research studied the effects of extensive versus moderate individual-item processing on the encoding of relational information. Of particular interest was the idea that excessive attention to individualitem information might interfere with discovering relations. In two studies, subjects performed an orienting task designed to induce either low or high individual-item processing for a list of words. Using clustering as our measure of relational processing, the results showed no difference in relational processing between the low and high individual-item processing groups. These results suggest that individual-item and relational processing are independent in the sense that increased individual-item processing neither enhances nor interferes with the encoding of relational information.
\end{abstract}

Memory researchers in the 1950 s and 1960 s tended to emphasize the importance of organization for memory (Mandler, 1967). The research was unanimous in showing that attention to relations among items provides large benefits for memory. Memory researchers in the 1970s, however, tended to emphasize the importance of semantic, elaborate (Craik \& Tulving, 1975), and eventually distinctive (Jacoby \& Craik, 1979) processing of individual items. The focus of this view was that attention to features that make items distinctive and discriminable from other items improves memory. More recent research acknowledges the benefits of both kinds of processing and has demonstrated that attention to both organizational and distinctive features produces better memory than either relational or individual-item processing alone (Einstein \& Hunt, 1980; Hunt \& Einstein, 1981).

Although a combination of both types of processing benefits memory, little research has studied whether increased processing of one type of information affects encoding of the other type of information. The present experiments were conducted to study the effects of extensive versus moderate individual-item processing on the encoding of relational information. It is generally thought that increased attention to features of items leads to a more elaborate or distinctive encoding and hence better memory. Better memory may not result, however, if this additional attention to item-specific features obscures the discovery of relations among items. To paraphrase a familiar adage, excessive attention to the trees could obscure perception of the forest.

M. J. Guynn is now in the Department of Psychology at Rice University. Address correspondence to Gilles O. Einstein, Department of Psychology, Furman University, Greenville, SC 29613.
A second possibility is that increased individual-item processing may lead to the encoding of more properties of items, which could provide the basis for discovering more relations among items and hence better memory. This idea is similar in some respects to the idea that increased elaboration leads to the development of more connections among items and hence more retrieval paths to the stored items (Bradshaw \& Anderson, 1982). Still a third possibility is that relational and individual-item processing are independent, so that increased individualitem processing has no effect on relational processing.

At present these views are difficult to evaluate because past studies for the most part have been designed to examine the effects of individual-item and relational processing on the encoding of item-specific and organizational information, respectively. Moreover, the few studies that have tested the effects of extensive individual-item processing on the encoding of relational information have produced equivocal results. For example, Einstein, McDaniel, Owen, and Cote (1990), using texts, found that increased individual-item processing beyond a moderate level actually decreased recall. They suggested that the lowered recall was due to the additional individual-item processing interfering with the encoding of relational information. Different results were obtained by McDaniel, Einstein, and Lollis (1988). Using word lists, they found that increased individual-item processing improved free recall and recognition scores. Increased individual-item processing did not, however, affect the encoding of relational information. In light of these conflicting results, the goal of the present research was to provide additional tests of how increased individual-item processing influences the encoding of relational information. 


\section{EXPERIMENT 1}

In the first experiment, the subjects were presented with a list of categorized target words and either one or three properties for each target item. The subjects were then instructed to rate how well the property(ies) described the target item. Our assumption was that rating three properties would produce more individual-item processing than rating one property. Because previous research has suggested that subjects tend to do less relational processing with incidental learning instructions (McDaniel \& Masson, 1977), we also varied whether subjects received incidental or intentional instructions.

\section{Method}

Design and Subjects. The design was a $2 \times 2 \times 2$ between-subject factorial, varying the amount of individual-item processing (low, high), mode of learning (incidental, intentional), and pacing of the orienting task (self-paced, experimenter-paced). The 12 subjects in each of the eight conditions were students enrolled in General Psychology at Furman University who received course credit for participating. The subjects were tested in small groups, and each session lasted $40 \mathrm{~min}$.

Materials and Procedure. The subjects were presented with 80 words, 1 word at a time, on an NCR computer screen. The list of words consisted of 4 words from each of 20 categories from the Battig and Montague (1969) category norms. Our aim was to construct a list in which the category relations were not too obvious. Thus, moderately frequent examples were selected from each category (i.e., items whose ranks in a category were between 5 and 20). The subjects in the low individualitem processing condition were presented with a target word followed by one property of that target (e.g., mansion-groundskeeper). The subjects in the high individual-item processing condition were presented with a target word followed by three properties of that target (e.g., mansion-rich, columns, groundskeeper). The properties were plausible associates of the target item that were not also associates of any other target item. All subjects were instructed to rate how well the property(ies) described each target word, on a scale of 1 to 5, with 1 being not descrip tive and 5 being very descriptive. Regardless of condition, the subjects made only one rating for each target item.

Half of the subjects were given incidental instructions. The others were assigned to the intentional condition, in which they were additionally instructed to try to remember the target words for a subsequent memory test. The subjects in the self-paced condition were allowed to perform the rating task at their own pace. The subjects in the experimenter-paced condition, who were tested after all subjects in the self-paced condition had been tested, were told that each target and property(ies) would appear on the screen for $8 \mathrm{sec}$ and that they would have as long as necessary to make their rating. The 8-sec rate was chosen because this was the average time taken by self-paced subjects in the incidental learning, three-property condition (the condition taking the longest amount of time).

After the words had been presented, the subjects were given a 2-min math-problem distractor task and then $10 \mathrm{~min}$ to recall the target words. The subjects were then given as much time as needed to complete a recognition test that consisted of the 80 target items randomly arranged with 80 new items (4 new items from each of the 20 categories in the target list).

\section{Results and Discussion}

Each of the memory measures was evaluated with a $2 \times 2 \times 2$ (individual-item processing condition $\times$ mode of learning $\times$ pacing of the orienting task) analysis of variance (ANOVA). For all analyses, the alpha level was set at .05 .

Free recall was measured by counting the number of words correctly recalled. The ANOVA of this measure indicated that free recall was reliably higher in the three- property $(M=25.75)$ than in the one-property $(M=$ 17.10) condition $\left[F(1,88)=37.04, M S_{\mathrm{e}}=48.43\right]$. No other main effects or interactions were significant $(F \mathrm{~s}<2.57)$.

The degree of relational processing was measured with Roenker, Thompson, and Brown's (1971) clustering measure, which ranges in value from -1.0 to $+1.0(+1.0$ indicates perfect clustering and 0 indicates chance clustering). The ANOVA indicated that clustering did not differ between the three-property $(M=.42)$ and the oneproperty $(M=.37)$ conditions $(F<1)$. The subjects in the experimenter-paced condition $(M=.46)$ had higher clustering than did the subjects in the self-paced condition $(M=.33)\left[F(1,88)=5.35, M S_{e}=.08\right]$. No other main effects or interactions were significant $(F \mathrm{~s}<1.93)$.

The degree of individual-item processing was measured by determining the probability of hits ( $p$ hit) and false alarms ( $p \mathrm{fa})$ on the recognition test and computing a recognition score with the formula: recognition = ( $p$ hit $-p \mathrm{fa}) /(1-p \mathrm{fa})$. Recognition performance is generally taken to be a measure of individual-item processing (Hunt \& Einstein, 1981) because information that can be used to discriminate among highly similar old and new items (i.e., item-specific information) is essential for good recognition performance. The ANOVA indicated that recognition scores were reliably higher in the three-property $(M=.91)$ than in the one-property $(M=.83)$ condition $\left[F(1,88)=11.09, M S_{\mathrm{e}}=.01\right]$. There was a marginally significant interaction between pacing of the orienting task and number of properties $[F(1,88)=4.00, p=.05]$. This interaction occurred because the subjects in the threeproperty condition had higher scores than did the subjects in the one-property condition only when the orienting task was self-paced $[F(1,88)=14.52]$. No other main effects or interactions were significant $(F \mathrm{~s}<1.80)$.

\section{EXPERIMENT 2}

The results of Experiment 1 indicated that although extensive individual-item processing increased free recall and recognition, it did not affect relational processing (as indexed by clustering scores). Perhaps the failure to find an effect of additional individual-item processing on the encoding of relational information was due to the nature of the elaboration induced by the high individual-item task. Presenting subjects with three properties for each target item may not have produced a more specific or distinctive encoding of the target item. Extensive individual-item processing might be more likely to interfere with relational processing when the item-specific information more precisely specifies an instance of each target item. We examined this possibility in Experiment 2 .

In Experiment 2, the subjects were presented with a list of categorized target words and either a general [e.g., The girl bought some __. (sandals)] or a distinctive [e.g., The college student purchased some brightly colored to wear with her outfit for her sorority's annual toga party. (sandals)] sentence frame for each target word. The sub- 
jects were instructed to rate how pleasant each target word was in the context of its sentence frame. Both general and distinctive sentences specified an instance of the target item, with the difference between the two conditions being that the distinctive sentences specified a more precise instance of the target. Our assumption was that distinctive elaboration, relative to general elaboration, would bias the encoding of features specific to each item.

We also varied the typicality of the target words in the present experiment. Relations among atypical instances are less easily detected (Greenberg \& Bjorklund, 1981) and may be more easily obscured by highly distinctive processing.

\section{Method}

Design and Subjects. The design was a $2 \times 2$ between-subject factorial, varying typicality of the target items (typical, atypical) and nature of the sentence frames (general, distinctive). The 12 subjects in each of the four conditions were students at Furman University who received $\$ 3$ or course credit for participating. The subjects were tested in small groups, and each session lasted $30 \mathrm{~min}$.

Materials and Procedure. All subjects were presented with the target words and sentence frames in booklets, with one target word and one sentence frame per page. The list of words consisted of three words from each of 12 categories from Gruenenfelder's (1981) typicality ratings. Half of the subjects received typical items, and half of the subjects received atypical items from the same category. Items were chosen so that typical and atypical items differed in overall typicality rating $\left[M s=1.72\right.$ and 2.86 , respectively; $\left.F(1,22)=34.51, M S_{e}=.23\right]$ but not in overall frequency rating $[M s=16.89$ and 16.17 , respectively; $\left.F(1,22)=.01, M S_{e}=304.24\right]$.

Although the target items were different in the typical and atypical conditions, an effort was made to use similar sentence frames in these two conditions. The subjects in the general and distinctive conditions received each target word with a general and distinctive sentence frame, respectively. All subjects were instructed to rate their impression of how pleasant the target word was in the context of the sentence, on a scale of 1 to 5 , with 1 meaning not pleasant and 5 meaning very pleasant. The subjects were allowed to work at their own pace.

After the words and sentences had been presented, the subjects were given a word-problem distractor task that lasted a minimum of $5 \mathrm{~min}$ and then $5 \mathrm{~min}$ to recall the target words. The subjects were then given $5 \mathrm{~min}$ to complete a recognition test that consisted of the 36 target items randomly arranged with 84 new items. The new items consisted of (1) the 36 items that had been targets for the group that differed in typicality, (2) 12 additional typical and 12 additional atypical items ( 1 typical and 1 atypical from each of the 12 categories in the target list), and (3) 24 additional distractors ( 2 typical and 2 atypical from each of 6 categories not represented in the target list). The distractors and targets did not differ in overall typicality or frequency ratings. The subjects in the typical and atypical conditions received the same recognition test, with targets for the typical group serving as distractors for the atypical group and vice versa.

\section{Results and Discussion}

A $2 \times 2$ (target typicality $\times$ sentence condition) ANOVA was performed for the recall, clustering, and recognition scores. Free recall was reliably higher for typical $(M=$ 12.96) than for atypical $(M=9.71)$ targets $[F(1,44)=$ $\left.12.41, M S_{\mathrm{e}}=10.22\right]$. There was no difference in free recall between the general $(M=11.13)$ and distinctive $(M=11.54)$ sentences $[F<1]$. The interaction of typicality and sentence-frame condition was not significant $[F(1,44)=1.60]$.

Clustering was reliably higher for typical $(M=.43)$ than for atypical $(M=.13)$ targets $\left[F(1,44)=5.39, M S_{e}\right.$
$=.20 \mathrm{~J}$. Importantly, there was no difference in clustering between the general $(M=.25)$ and distinctive $(M=$ .32) sentence conditions $(F<1)$. The interaction of typicality and sentence-frame condition was not significant $(F<1)$.

Recognition was not reliably different between the typical $(M=.89)$ and atypical $(M=.92)$ conditions $[F(1,44)$ $\left.=1.56, M S_{\mathrm{e}}=.01\right]$ or between the general $(M=.91)$ and distinctive $(M=.90)$ sentence conditions $(F<1)$, and the interaction between these two variables was not significant $(F<1)$. Because recognition performance was very high (hits ranged from $89 \%$ to $93 \%$ ], ceiling effects may have obscured any differences between the two sentence conditions. Therefore, 36 of the 48 subjects were given a second recognition test 13-29 days later. Performance on the second recognition test was well below ceiling. Recognition scores were nominally higher for the distinctive condition $(M=.35)$ than for the general condition $(M=.26)$; however, none of the effects in this ANOVA were reliable $(F \mathrm{~s}<2.40)$.

In summary, these results showed that a task designed to promote more specific processing of individual items had no effect on relational processing. This interpretation is qualified, however, by our failure to find a significant effect of distinctive sentence frames on recognition performance (i.e., a measure of individual-item information).

\section{GENERAL DISCUSSION}

Our results showed that manipulations designed to enhance processing of item-specific information did not produce differences in clustering of items from categorized word lists. This occurred when subjects rated properties of the items (Experiment 1) and when subjects rated the items in the context of sentence frames (Experiment 2). It appears, therefore, that extra attention to the trees neither obscures nor enhances perception of the forest.

Perhaps extensive individual-item processing does not obscure taxonomic relations because these kinds of relations are so easily detected. Taxonomic categories generally are quite familiar to adult subjects. Less familiar, ad hoc categories do not show spontaneous relational processing when subjects are oriented to individual items (Einstein \& Hunt, 1980). Thus, the present results would appear to be due to the familiarity of the taxonomic categories used in this experiment.

A question of considerable interest for future research concerns the processing of other types of relationships. For example, are causal relations affected by item-specific processing in the same way as taxonomic relations? Einstein et al. (1990) discovered that increased individualitem processing of text decreased recall, perhaps because of disrupted relational processing. Relations in textual materials may be more easily obscured by individual-item processing. Inasmuch as often-used study strategies, such as notetaking, highlighting, and answering embedded questions, seem to draw attention primarily to individualitem information, further research on this issue has important educational implications. 


\section{REFERENCES}

Battig, W. F., Montague, W. E. (1969). Category norms for verbal items in 56 categories: A replication and extension of the Connecticut category norms. Journal of Experimental Psychology Monograph, 80(3, Pt. 2).

Bradshaw, G. L., \& ANDerson, J. R. (1982). Elaborative encoding as an explanation of levels of processing. Journal of Verbal Learning \& Verbal Behavior, 21, 165-174.

Craik, F. I. M., \& Tulving, E. (1975). Depth of processing and the retention of words in episodic memory. Journal of Experimental Psychology: General, 104, 268-294.

EINSTEIN, G. O., \& HUNT, R. R. (1980). Levels of processing and organization: Additive effects of individual-item and relational processing. Joumal of Experimental Psychology: Human Learning \& Memory, 6, 588-598.

Einstein, G. O., McDaniel, M. A., Owen, P. D., \& Coté, N. C. (1990). Encoding and recall of texts: The importance of material appropriate processing. Journal of Memory \& Language, 29, 566-581.

Greenberg, M. S., \& BJoRKLUND, D. F. (1981). Category typicality in free recall: Effects of feature overlap or differential category encoding? Journal of Experimental Psychology: Human Learning \& Memory, 7, 145-147.

GRUENENFELDER, T. M. (1981). Accessing semantic features and log- ical relations in category verification. Unpublished doctoral dissertation, Indiana University, Bloomington, IN.

Hunt, R. R., \& EINSTEIN, G. O. (1981). Relational and item-specific information in memory. Journal of Verbal Learning and Verbal Behavior, 20, 497-514.

JACOBY, L. L., \& CRAIK, F. I. M. (1979). Effects of elaboration of processing at encoding and retrieval: Trace distinctiveness and recovery of initial context. In L. S. Cermak \& F. I. M. Craik (Eds.), Levels of processing in human memory (pp. 1-21). Hillsdale, NJ: Erlbaum.

MANDleR, G. (1967). Organization and memory. In K. W. Spence \& J. T. Spence (Eds.), The psychology of learning and motivation (Vol. 1, pp. 327-372). New York: Academic Press.

MCDaniel, M. A., Einstein, G. O., \& Lollis, T. (1988). Qualitative and quantitative considerations in encoding difficulty effects. Memory \& Cognition, 16, 8-14.

McDaniel, M. A., \& MAsson, M. E. (1977). Long-term retention: When incidental semantic processing fails. Journal of Experimental Psychology: Human Learning \& Memory, 3, 270-281.

Roenker, D. L., Thompson, C. P., \& Brown, S. C. (1971). Comparison of measures for the estimation of clustering in free recall. Psychological Bulletin, 76, 45-48.

(Manuscript received October 7, 1991.) 